\title{
On Invariants of Graphs Related to Quantum sl(2) at Roots of Unity
}

\author{
NATHAN GEER ${ }^{1,2}$ and NICOLAI RESHETIKHIN ${ }^{3,4}$ \\ ${ }^{1}$ Max-Planck Institute für Mathematik, Vivatgasse 7, 53111 Bonn, Germany. \\ ${ }^{2}$ School of Mathematics, Georgia Institute of Technology, Atlanta, GA 30332-0160, USA. \\ e-mail: geer@math.gatech.edu \\ ${ }^{3}$ Department of Mathematics, University of California, Berkeley, 970 Evans Hall \#3840, \\ Berkeley, CA 94720-3840, USA. \\ ${ }^{4}$ KDV Institute for Mathematics, Universiteit van Amsterdam, Plantage Muidergracht 24, \\ 1018 TV, Amsterdam, The Netherlands.e-mail: reshetik@math.berkeley.edu
}

Received: 14 March 2009 / Accepted: 4 April 2009

Published online: 13 May 2009 - (C) The Author(s) 2009. This article is published with open access at Springerlink.com

\begin{abstract}
We show how to define invariants of graphs related to quantum $s l_{2}$ when the graph has more then one connected component and components are colored by blocks of representations with zero quantum dimensions.
\end{abstract}

Mathematics Subject Classification (2000). 17B37, 57R56, 46A63.

Keywords. invariants of knots, quantized universal enveloping algebras, topological quantum field theory.

\section{Introduction}

The invariants of oriented linked framed graphs colored by finite dimensional representations of quantized universal enveloping algebras were constructed in [4] for non-zero quantum dimensions. In this construction the edges of the graph are colored by finite dimensional representations. At each vertex a total ordering of adjacent edges which agrees with counter-clockwise cyclic order defined by the framing is fixed. A vertex with adjacent edges colored by representations $V_{1}, \ldots, V_{n}$ (according to the total ordering) is colored by an element from $\operatorname{Hom}\left(\mathbb{C}, V_{1}^{\epsilon_{1}} \otimes \ldots \otimes V_{n}^{\epsilon_{n}}\right)$ of all $U_{q}\left(s l_{2}\right)$-invariant vectors in the tensor product. Here $V^{+}=V, V^{-}=V^{*}$ where $V^{*}$ is the left dual to $V, \epsilon=+$ if the edges is incoming and $\epsilon=-$ if the edges is outgoing.

When the quantum dimension is zero, it is easy to define such invariants for knots when the center of $U_{q}\left(s l_{2}\right)$ acts by scalars on the representation coloring the knot. One should cut the knot (its diagram) at any place and then to compute the invariant of the corresponding $(1,1)$ tangle. Because the center acts trivially at the coloring representation, this invariant will be equal to a operator of multiplication by 
a scalar. It is easy to show that the scalar is the invariant of a knot (does not depend on where the knot was cut). But this technique does not work for links.

Recently invariants of links colored by such representations were introduced in [2] under the assumption that these representations satisfy an additional property, i.e. they are ambidextrous (see [2] or Section 3.1 for the precise definition). We will abbreviate this name here to ambi-modules.

The goal of this note is to present in details how the construction involving ambi-modules works for $s l_{2}$. We prove that all generic irreducible representations of $\stackrel{\circ}{U}_{\epsilon}\left(s l_{2}\right)$ are ambi-modules.

We also conjecture but we do not prove it here that all generic irreducible representations of the $\stackrel{\circ}{U}_{\epsilon}(\mathfrak{g})$ are ambi-modules for any simple Lie algebra.

We would like to thank V. Turaev and B. Patureau for discussions. This work was supported by the Danish National Research Foundation through the Niels Bohr initiative, the work of N.R. was also supported by the NSF grant DMS0601912. The work of N.G. was supported by the NSF grant DMS-0706725. Both authors are grateful to the Aarhus University for the hospitality.

\section{The Split Form of Quantum $s l_{2}$ at a Root of Unity}

\subsection{QUANTUM $s l_{2}$}

Let $m, l$ be positive odd integers, $t=\exp \left(\frac{i \pi m}{2 l}\right)$, and $\epsilon=t^{2}$.

The algebra $U_{\epsilon}\left(s l_{2}\right)$ is the unital $\mathbb{C}$-algebra generated by invertible $K$ and by $E, F$ with defining relations:

$$
K E=\epsilon^{2} E K, \quad K F=\epsilon^{-2} F K, \quad[E, F]=\frac{K-K^{-1}}{\epsilon-\epsilon^{-1}} .
$$

Recall that it is a Hopf algebra with the comultiplication acting on generators as

$$
\Delta K=K \otimes K, \quad \Delta E=E \otimes K+1 \otimes E, \quad \Delta F=F \otimes 1+K^{-1} \otimes F
$$

The elements $K^{l}, F^{l}, E^{l}$ generate a central Hopf subalgebra, and $E^{l}, F^{l}$ generate central Hopf ideal. Denote by $U_{\epsilon}\left(s l_{2}\right)^{\prime}$ its quotient by this central ideal.

\subsection{THE SPLIT FORM OF $U_{\epsilon}\left(s l_{2}\right)^{\prime}$}

DEFINITION 1. The split form of $U_{\epsilon}\left(s l_{2}\right)^{\prime}$ is the algebra $\stackrel{\circ}{U}_{\epsilon}\left(s l_{2}\right)$ generated by $P_{i}, i \in \mathbb{Z}$ and by $E, F, x, x^{-1}$ such that $x$ is central and

$$
\begin{aligned}
& P_{i} P_{j}=\delta_{i j} P_{i}, \quad P_{i} E=E P_{i-2}, \quad P_{i} F=F P_{i+2}, \quad \sum_{i \in \mathbb{Z}} P_{i}=1, \\
& E F-F E=\sum_{j \in \mathbb{Z}} \frac{x^{2} \epsilon^{j}-x^{-2} \epsilon^{-j}}{\epsilon-\epsilon^{-1}} P_{j} .
\end{aligned}
$$


The algebra $\stackrel{\circ}{U}_{\epsilon}\left(s l_{2}\right)$ is a Hopf algebra (in the category of its finite-dimensional representations) where the comultiplication $\Delta$, counit $\varepsilon$ and antipode $S$ morphisms are given by:

$$
\begin{aligned}
\Delta(E) & =\sum_{j \in \mathbb{Z}} E \otimes x^{2} \epsilon^{j} P_{j}+1 \otimes E, \quad \Delta(F)=F \otimes 1+\sum_{j \in \mathbb{Z}} x^{-2} \epsilon^{-j} P_{j} \otimes F, \\
\Delta\left(P_{k}\right) & =\sum_{i, j \in \mathbb{Z} ; i+j=k} P_{i} \otimes P_{j}, \quad \Delta(x)=x \otimes x, \\
\varepsilon(E) & =\varepsilon(F)=0, \quad \varepsilon\left(P_{i}\right)=\delta_{i, 0}, \quad \varepsilon(x)=1, \\
S(E) & =-E \sum_{j \in \mathbb{Z}} x^{-2} \epsilon^{-j} P_{j}, \quad S(F)=-\sum_{j \in \mathbb{Z}} x^{2} \epsilon^{j} P_{j} F, \quad S\left(P_{i}\right)=P_{-i}, \quad S(x)=x^{-1} .
\end{aligned}
$$

It is clear that the map $\iota: U_{\epsilon}\left(s l_{2}\right) \rightarrow \stackrel{\circ}{U_{\epsilon}}\left(s l_{2}\right)$ given by $K \mapsto \sum_{j \in \mathbb{Z}} x^{2} \epsilon^{j} P_{j}, E \mapsto E$ and $F \mapsto F$ is an injective morphism of Hopf algebras.

Notice that $K^{l} \mapsto \sum_{j \in \mathbb{Z}} x^{2 l} \epsilon^{j l} P_{j}$ and that $K^{2 l} \mapsto x^{4 l}$.

\subsection{THE $R$-MATRIX}

For $n \in \mathbb{N}$ we set $[n]=\frac{\epsilon^{n}-\epsilon^{-n}}{\epsilon-\epsilon^{-1}}$ and $[n] !=[n][n-1] \ldots[1]$. Define

$$
\begin{aligned}
& R_{0}=\sum_{i, j \in \mathbb{Z}} t^{i j} x^{i} P_{j} \otimes x^{j} P_{i}, \\
& R_{1}=\sum_{n=0}^{l-1} \frac{\left(\epsilon-\epsilon^{-1}\right)^{n}}{[n] !} E^{n} \otimes F^{n},
\end{aligned}
$$

and $R=R_{0} R_{1} \in \stackrel{\circ}{U_{\epsilon}}\left(s l_{2}\right)^{\otimes 2}$.

Now we will show that $R$ defines a quasitriangular structure on $\stackrel{\circ}{U}_{\epsilon}\left(s l_{2}\right)$.

LEMMA 2. The element $R_{0}$ satisfies the following identities.

(1) $(\Delta \otimes \mathrm{Id})\left(R_{0}\right)=\left(R_{0}\right)_{13}\left(R_{0}\right)_{23}$,

(2) $(\mathrm{Id} \otimes \Delta)\left(R_{0}\right)=\left(R_{0}\right)_{13}\left(R_{0}\right)_{12}$,

(3) $R_{0}(E \otimes 1)=(E \otimes \iota(K)) R_{0}, \quad R_{0}(1 \otimes E)=(\iota(K) \otimes E) R_{0}$,

(4) $R_{0}(F \otimes 1)=\left(F \otimes \iota(K)^{-1}\right) R_{0}, R_{0}(1 \otimes F)=\left(\iota(K)^{-1} \otimes F\right) R_{0}$.

Proof. We will prove the first identity in Part (3), the other identities follow similarly. We have

$$
\begin{aligned}
R_{0}(E \otimes 1) & =\sum_{i, j \in \mathbb{Z}} t^{i j} x^{i} P_{j} E \otimes x^{j} P_{i}= \\
& =\sum_{i, j \in \mathbb{Z}} t^{i j} x^{i} E P_{j-2} \otimes x^{j} P_{i}=
\end{aligned}
$$




$$
\begin{aligned}
& =\sum_{i, j \in \mathbb{Z}} t^{i j} x^{i} E P_{j} \otimes x^{j} x^{2} \epsilon^{i} P_{i}= \\
& =(E \otimes \iota(K)) R_{0} .
\end{aligned}
$$

THEOREM 3. The pair $\left(\stackrel{\circ}{U}_{\epsilon}\left(s l_{2}\right), R\right)$ is a quasitriangular Hopf algebra.

Proof. Lemma 2 implies that $R_{0}$ has the same commutator relation with $E$ and $F$ as the element $\exp \left(\frac{h}{4}(H \otimes H)\right)$ in the $\mathbb{C}[[h]]$-algebra $U_{h}\left(s l_{2}\right)$. Thus, we conclude

$$
(\Delta \otimes \mathrm{Id})(R)=R_{13} R_{23}, \quad(\operatorname{Id} \otimes \Delta)(R)=R_{13} R_{12},
$$

and

$$
\Delta^{o p}(a)=R \Delta(a) R^{-1}, \text { for } a \in \stackrel{\circ}{U}_{\epsilon}\left(s l_{2}\right)
$$

where $\Delta^{o p}=\tau \circ \Delta$ and $\tau$ is the permutation $a \otimes b \mapsto b \otimes a$.

Let $R=\sum s_{i} \otimes t_{i}$ and define $u=\sum S\left(t_{i}\right) s_{i}$. Then following [1] we have

$$
\epsilon(u)=1, \quad \Delta(u)=\left(R_{21} R\right)^{-1}(u \otimes u), \quad S^{2}(a)=u a u^{-1}
$$

for all $a \in \stackrel{\circ}{U}_{\epsilon}\left(s l_{2}\right)$.

A direct computation shows that for all $a \in \stackrel{\circ}{U}_{\epsilon}\left(s l_{2}\right)$ we have

$$
S^{2}(a)=\iota(K) a \iota(K)^{-1} .
$$

Let $\tilde{\theta}=u \iota(K)^{-1}=\iota(K)^{-1} u$. Comparing this formula with the formula for the $S^{2}$ involving $u$ we see that $\tilde{\theta}$ is central.

LEMMA 4. The element $\tilde{\theta}$ satisfies the relations:

$$
\epsilon(\tilde{\theta})=1, \quad \Delta(\tilde{\theta})=\left(R_{21} R\right)^{-1}(\tilde{\theta} \otimes \tilde{\theta}), \quad S(\tilde{\theta})=\iota(K)^{2 l} \tilde{\theta} .
$$

Proof. The first two relations follow from (1) and the definition of the counit and coproduct of $\iota(K)$. The last relation can be proven by direct computation of $S\left(u \iota(K)^{-1}\right)$ and of $u \iota(K)^{-1}$. This computation was done essentially in [3]. One can also prove the last identity by computing how $S(\tilde{\theta})$ and $\tilde{\theta}$ act on generic irreducible modules. It is easy to see that on these modules the identity holds. On the other hand our algebra is finitely generated and is finite dimensional over the center. This implies that the identity $S(\tilde{\theta})=\iota(K)^{2 l} \tilde{\theta}$ holds not only for generic points but also for special points (where $x$ acts as $4 l$ th root of unity). 


\subsection{THE AUTOMORPHISM $\phi$}

PROPOSITION 5. (1) The mapping $\phi: \stackrel{\circ}{U_{\epsilon}}\left(s l_{2}\right) \rightarrow \stackrel{\circ}{U}_{\epsilon}\left(s l_{2}\right)$ acting on generators as

$$
\phi\left(P_{i}\right)=P_{i+2}, \quad \phi(x)=x \epsilon, \quad \phi(E)=E, \quad \phi(F)=F
$$

extends uniquely to an algebra automorphism.

(2) The set of fixed points form a Hopf subalgebra generated by $x \sum_{i \in \mathbb{Z}} t^{i} P_{i}, x^{2 l}$, $E, F$.

Proof. The first part is clear from defining relations, the second follows immediately from the formula for $\phi$.

Note that despite the fact that fixed points of $\phi$ form a Hopf subalgebra, it is not a Hopf algebra automorphism.

The automorphism $\phi$ acts on the $R$-matrix as follows:

$$
(\phi \otimes i d)(R)=\left(1 \otimes x^{-2}\right) R, \quad(i d \otimes \phi)(R)=\left(x^{-2} \otimes 1\right)(R)
$$

PROPOSITION 6. The automorphism $\phi$ acts on $\tilde{\theta}$ as follows:

$$
\phi(\tilde{\theta})=x^{4} \tilde{\theta}
$$

Proof. Let $R=\sum_{i} s_{i} \otimes t_{i}$, then $R^{-1}=\sum_{i} s_{i} \otimes S\left(t_{i}\right)$. Combining the way $\phi$ acts on the $R$-matrix with the fact that it is an algebra automorphism we obtain $(\phi \otimes$ $\phi)\left(R^{-1}\right)=\left(x^{2} \otimes x^{2}\right) R^{-1}$. Therefore $\phi\left(\sum_{i} S\left(t_{i}\right) s_{i}\right)=x^{4} \sum_{i} S\left(t_{i}\right) s_{i}$. Thus, $\phi(u)=x^{4} u$. Since $\phi(\iota(K))=\iota(K)$, this proves the proposition.

Define $\varepsilon=\sum_{j \in \mathbb{Z}}(-1)^{j} P_{j}$. It is central and unipotent. It is also easy to see that $\phi$ acts trivially on it:

$$
\phi(\varepsilon)=\varepsilon
$$

\subsection{THE EXTENDED $R$-MATRIX}

Consider the algebra $A_{\epsilon}=\stackrel{\circ}{U}_{\epsilon}\left(s l_{2}\right) \otimes_{\mathbb{C}} \mathbb{C}\left[\tau, \tau^{-1}\right]$. The element $\tau$ is central in this algebra.

Remark 7. The motivation for this construction is the formal power series version of the algebra $\stackrel{\circ}{U}_{\epsilon}\left(s l_{2}\right)$, when instead of having Laurent polynomials in $x$ we have formal powers series $\mathrm{z}$ :

$$
x=\exp \left(\frac{i m z \pi}{2 l}\right), \quad \tau=\exp \left(\frac{i m z^{2} \pi}{4 l}\right) .
$$


Assume that the action of the comultiplication on $\tau$ is symmetric, i.e. $\Delta^{o p}(\tau)=$ $\Delta(\tau)$. Define

$$
\rho=\Delta(\tau) \tau^{-1} \otimes \tau^{-1}
$$

Extend the action of the automorphism $\phi$ on $A_{\epsilon}$ as:

$$
\phi(\tau)=\epsilon x^{2} \tau, \quad(\phi \otimes i d)(\rho)=\left(1 \otimes x^{2}\right) \rho .
$$

Define the extended $R$-matrix as

$$
\mathcal{R}=\rho R
$$

It is easy to see that $\mathcal{R}$ defines a quasitriangular structure on $A_{\epsilon}$. It is also easy to see that

$$
(i d \otimes \phi)(\mathcal{R})=(\phi \otimes i d)(\mathcal{R})=\mathcal{R} .
$$

Define

$$
\theta_{1}=\tau^{-2} \iota(K)^{l} \tilde{\theta}, \quad \theta_{2}=\tau^{-2} \iota(K)^{l} \varepsilon \tilde{\theta} .
$$

Each of these elements satisfies identities:

$$
\epsilon(\theta)=1, \quad \Delta(\theta)=\left(\mathcal{R}_{21} \mathcal{R}\right)^{-1}(\theta \otimes \theta), \quad S(\theta)=\theta, \quad \phi(\theta)=\theta .
$$

\section{The Category $C_{\epsilon}$}

\subsection{SIMPLE MODULES}

Here we will focus on simple modules over the $\stackrel{\circ}{U}_{\epsilon}\left(s l_{2}\right), U_{\epsilon}\left(s l_{2}\right)^{\prime}$, and $A_{\epsilon}$. We will say $a \in \mathbb{C}^{*}$ is generic if $a^{4 l} \neq 1$.

The following statement is a variation on a well known fact:

PROPOSITION 8. For each generic $a \in \mathbb{C}^{*}$ and $k \in \mathbb{Z}$ there exists unique simple $\stackrel{\circ}{U}_{\epsilon}$ $\left(s l_{2}\right)$-module $V(a, k)$ with highest weight vector $v_{k}$ such that

$$
E v_{k}=0, \quad x v_{k}=a v_{k} \quad P_{j} v_{k}=\delta_{j, k} v_{k}, \quad V(a, k)=\sum_{i=0}^{l-1} \mathbb{C} F^{i} v_{k} .
$$

It is easy to compute the action of generators on the weight basis $v_{k-2 j}=F^{j} v_{k}$ :

$$
\begin{aligned}
x v_{k-2 i} & =a v_{k-2 i} \\
P_{j} v_{k-2 i} & =\delta_{j, k-2 i} v_{k-2 i}, \quad 0 \geq i \geq l-1, \\
F v_{k-2 i} & =v_{k-2 i-2}, \quad 1 \leq i \leq l-1 \\
E v_{k-2 i} & =\left(\frac{a^{2} \epsilon^{k+i+1}-a^{-2} \epsilon^{-k-1-i}}{\epsilon-\epsilon^{-1}}\right)\left(\frac{\epsilon^{i}-\epsilon^{-i}}{\epsilon-\epsilon^{-1}}\right) v_{k-2 i+2}, \quad 0 \leq i \leq l-1 .
\end{aligned}
$$


The homomorphism $\iota: U_{\epsilon}\left(s l_{2}\right)^{\prime} \rightarrow \stackrel{\circ}{U}_{\epsilon}\left(s l_{2}\right)$ defines on $V(a, k)$ the structure of a $U_{\epsilon}\left(s l_{2}\right)^{\prime}$-module.

PROPOSITION 9. The $U_{\epsilon}\left(s l_{2}\right)^{\prime}$-modules $V(a, k)$ are irreducible, and in addition, $V(a, k)$ and $V(a \epsilon, k+1)$ are isomorphic as $U_{\epsilon}^{\prime}$-modules.

In particular central element $\varepsilon$ acts on $V(a, k)$ as $(-1)^{k}$.

Note that the modules $V(a, k)$ and $V(a \epsilon, k+1)$ are not isomorphic as $\stackrel{\circ}{U}\left(s l_{2}\right)$ modules.

For $z \in \mathbb{C}$ we can always define the $A_{\epsilon}$-module structure on the representation $V\left(e^{\frac{i m z \pi}{2 l}}, k\right)$ of $\stackrel{\circ}{U}_{\epsilon}\left(s l_{2}\right)$ by defining $\tau v=\exp \left(\frac{i m z^{2} \pi}{4 l}\right) v$ for any $v \in V\left(e^{\frac{i m z \pi}{2 l}}, k\right)$. It is clear that this defines an irreducible $A_{\epsilon}$-module.

\subsection{THE CATEGORY $C_{\epsilon}$}

Objects of the category $C_{\epsilon}$ are finite dimensional $\stackrel{\circ}{U}_{\epsilon}\left(s l_{2}\right)$-modules $\left(V, \pi_{V}: A_{\epsilon} \rightarrow\right.$ $\operatorname{End}(V))$ on which $x$ acts as a multiplication by a scalar $\pi_{V}(x) v=\exp \left(\frac{i m z \pi}{2 l}\right) v$. Here $v \in V, m, l$ are as above and $z \in \mathbb{C}$. The central element $\tau$ on such module acts by multiplication on $\exp \left(\frac{i m z^{2} \pi}{4 l}\right)$.

Morphisms between two such modules are all $\stackrel{\circ}{U}_{\epsilon}\left(s l_{2}\right)$-invariant linear maps.

This category is monoidal because it is a category of finite dimensional modules over a Hopf algebra. It is a rigid monoidal category with the left dual modules defined as usual $\left(V^{*}, \pi_{V^{*}}=\pi_{V}^{*} \circ S\right)$ where $\pi_{V}^{*}(a)$ is the dual linear map to $\pi_{V}(a)$ and with usual injection and evaluation morphisms:

$$
\begin{gathered}
i_{V}: \mathbb{C} \rightarrow V \otimes V^{*}, \text { given by } 1 \mapsto \sum e_{i} \otimes e^{i}, \\
e_{V}: V^{*} \otimes V \rightarrow \mathbb{C}, \text { given by } f \otimes w \mapsto f(w)
\end{gathered}
$$

It is easy to see that $C_{\epsilon}$ is a braided category with the commutativity morphism $c=\left\{c_{V, W}\right\}$ where $c_{V, W}: V \otimes W \rightarrow W \otimes V$ given by $v \otimes w \mapsto \tau(R(v \otimes w))$. It is also a ribbon category with the ribbon morphisms (twists): $\theta_{V}: V \rightarrow V, v \mapsto \theta^{-1} v$.

In this category the braiding and the ribbon structure agree with isomorphisms of modules induced by $\phi$.

The objects of this category are semisimple for generic $z$, i.e. when $m z$ it is not an integer.

The linear mapping $v \rightarrow \theta^{-1} u v$ is a an isomorphism of representations $V \rightarrow V^{* *}$. Recall that the quantum (functorial) dimension of $V$ is defined as the composition mapping $\mathbb{C} \rightarrow V \otimes V^{*} \rightarrow V^{* *} \otimes V^{*} \rightarrow \mathbb{C}$, or, as $\operatorname{tr}_{V}\left(\theta^{-1} u\right)=\operatorname{tr}_{V}\left(\tau^{2} \iota(K)^{1-l}\right)$. It is clear that the quantum dimension of any generic representation is zero. 


\section{Invariants of Links}

\subsection{AMBI- ELEMENTS IN A RIBBON CATEGORY}

Here we will recall some results and definitions from [2].

Recall that in a ribbon category there is a natural notion of a trace of an endomorphism of an object. If $f: V \rightarrow V$, its trace is

$$
\operatorname{tr}_{V}(f)=e_{V^{*}} \circ\left(\mu_{V} \otimes i d_{V^{*}}\right) \circ\left(f \otimes i d_{V^{*}}\right) \circ i_{V}
$$

where $\mu_{V}: V \rightarrow V^{* *}$ is the isomorphism between $V$ and $V^{* *}$ determined by the braiding and the ribbon structure. For the category of $\stackrel{\circ}{U}_{\epsilon}\left(s l_{2}\right), \mu_{V}=\pi_{V}\left(\iota(K)^{1-l}\right)$.

We will use the following notations. If $V$ is simple and $f: V \rightarrow V$ is a morphism, by definition $f=c(f) i d_{V}$ for some $c(f) \in \kappa$ where $\kappa$ is the base field for our category. We will assume $\kappa=\mathbb{C}$ and will use the graphical notation for $c(f)$ shown on Figure 1.

Define $S^{\prime}(U, V)=\left(i d_{V} \otimes \operatorname{tr}_{U}\right)\left(c_{U, V} c_{V, U}\right)$ where $c_{V, U}: V \otimes U \rightarrow U \otimes V$ is the commutativity constraint in the category. This element can be written graphically as on Figure 2.

For any $f: V \rightarrow V$ and simple $V$ we have the identity shown on Figure 3.

Recall that an object $V$ is called ambidextrous if $\left(i d_{V} \otimes \operatorname{tr}_{V}\right)(f)=\left(\operatorname{tr}_{V} \otimes i d_{V}\right)(f)$ for any $f: V^{\otimes 2} \rightarrow V^{\otimes 2}$ (see also Figure 4). Here we will call such an object an ambi-object.

The following observation is one of the key tools from [2]. Let $U$ be a simple ambi-object. Then for any two objects $V_{1}$ and $V_{2}$ and any morphism $f: V_{1} \otimes V_{2} \rightarrow$ $V_{1} \otimes V_{2}$ the following identity holds:

$$
S^{\prime}\left(U^{*}, V_{1}^{*}\right) S^{\prime}\left(V_{2}, U\right) c\left(\left(\operatorname{tr}_{V_{1}} \otimes i d_{V_{2}}\right)(f)\right)=S^{\prime}\left(V_{1}, U\right) S^{\prime}\left(U^{*}, V_{2}^{*}\right) c\left(\left(i d_{V_{1}} \otimes \operatorname{tr}_{V_{2}}\right)(f)\right)
$$

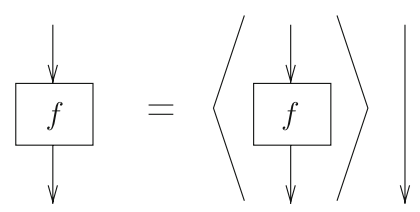

Figure 1.

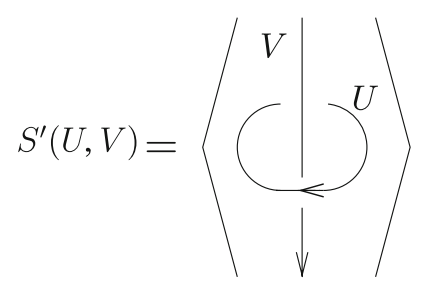

Figure 2. 


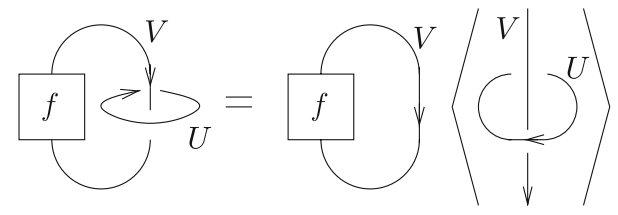

Figure 3.

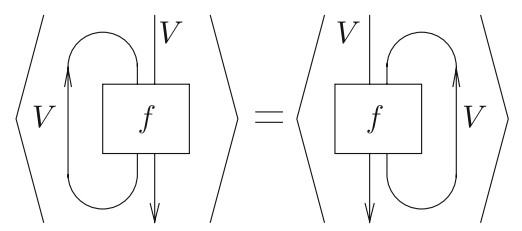

Figure 4.

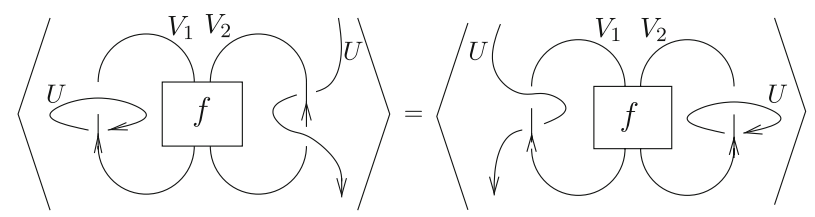

Figure 5.

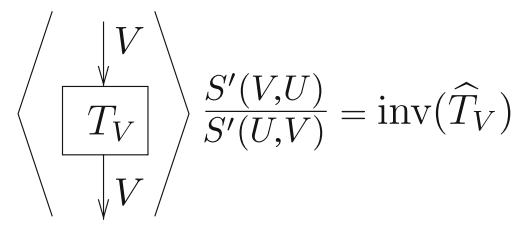

Figure 6. $\widehat{T}_{V}$ is the closer of $T_{V}$.

It follows from the ambi-identity for the tangle from Figure 5. Here notice that $S^{\prime}\left(V^{*}, W^{*}\right)=S^{\prime}(V, W)$.

We have the following corollary of the identity (3).

PROPOSITION 10. - If $J$ is a simple ambi-object of the category $\mathbb{C}$, then all objects $U \in A(J)=\left\{W \mid S^{\prime}(W, J) \neq 0, S^{\prime}(J, W) \neq 0, W\right.$ is simple $\}$ are also ambiobjects.

- Let $T_{V}$ be a $(1,1)$-tangle colored by elements of $\mathcal{C}_{\epsilon}$, whose open component is colored by an element $V \in A(J)$. Then the expression in Figure 6 is an invariant of the link obtained by the closure of $T_{V}$. In this expression $U$ is any ambi-element from $A(J)$. 


\subsection{AMBI- MODULES OVER $\stackrel{\circ}{U}_{\epsilon}\left(s l_{2}\right)$}

3.2.1. As it was pointed out at the end of the previous section all modules $V(a, k)$ have zero quantum dimension. Here we will prove that they are all ambi-objects in the category $\stackrel{\circ}{U}_{\epsilon}\left(s l_{2}\right)-\bmod$.

LEMMA 11. The module $V(a, k)$ is an ambi-object for all $k \in \mathbb{Z}$ and $a^{8 l} \neq 1$.

Proof. It is easy to prove that for all $a \in \mathbb{C} \backslash\left\{a^{8 l}=1\right\}$, and $k \in \mathbb{Z}$

$$
V(a, k) \otimes V(a, k) \cong V(2 a, 2 k) \oplus V(2 a, 2 k-2) \oplus \cdots \oplus V(2 a, 2 k-2 l+2) .
$$

Since this decomposition is a direct sum of non-isomorphic simple modules the algebra $\operatorname{End}(V(a, k) \otimes V(a, k))$ is commutative. Now let $f \in \operatorname{End}(V \otimes V)$, then $\left(i d_{V} \otimes \operatorname{tr}_{V}\right)(f)=\left(\operatorname{tr}_{V} \otimes i d_{V}\right)\left(c_{V, V}^{-1} \circ f \circ c_{V, V}\right)$. But $c_{V, V}$ commutes with $\operatorname{End}(V \otimes V)$ and so $c_{V, V}^{-1} \circ f \circ c_{V, V}=f$. Here we set $V=V(a, k)$. This finishes the proof.

The condition $a^{8 l} \neq 1$ can be relaxed to $a^{4 l} \neq 1$.

LEMMA 12. Let $V(a, k)$ and $V(b, m)$ be two irreducible $\stackrel{\circ}{U}_{\epsilon}\left(s l_{2}\right)$-modules described in the previous section. Then

$$
\begin{aligned}
S^{\prime}(V(a, k), V(b, m))= & \epsilon^{(k+1-l)(m+1-l)} b^{2 k-2 l+2} a^{2 m-2 l+2}(-1)^{m+l-1} \times \\
& \times\left[\frac{b^{2 l}-b^{-2 l}}{\epsilon^{m+1-l} b^{2}-\left(\epsilon^{m+1-l} b^{2}\right)^{-1}}\right]
\end{aligned}
$$

Proof. The proof is a straightforward computation. We want to compute the action of $\left(i d_{V} \otimes \operatorname{tr}_{U}\right)\left(\left(\pi_{V} \otimes \pi_{U}\right)(\sigma(R) R)\right)$ on $V$. Let us apply to the highest weight vector. Then

$$
\begin{aligned}
S^{\prime}\left((V(a, k), V(b, m)) v_{m}\right. & =\left(i d_{V(b, m)} \otimes \operatorname{tr}_{V(a, k)}\right)\left(\left(\pi_{V(b, m)} \otimes \pi_{V(a, k)}\right)\left(\sigma\left(R_{0}\right) R_{0}\right)\right) v_{m}= \\
& =\epsilon^{k(m+1-l)} b^{2 k} a^{2 m+2-2 l} \sum_{i=0}^{l-1} \epsilon^{-2 i(m+1-l)} b^{-4 i} v_{m}
\end{aligned}
$$

Then one should sum up the geometric progression.

Now, note that $S^{\prime}(V(a, k), V(b, m)) \neq 0$ unless $b^{4 l}=1$ when $\left(\epsilon^{m+1-l} b^{2}\right)^{2} \neq 1$. In the later case the representation $V(b, m)$ is reducible. We assume that this is not the case.

Thus, we have proven the following theorem.

THEOREM 13. All irreducible representations $V(a, k)$ of $\stackrel{\circ}{U}_{\epsilon}\left(s l_{2}\right)$ with $a^{4 l} \neq 1$ are ambi-modules. 
Open Access This article is distributed under the terms of the Creative Commons Attribution Noncommercial License which permits any noncommercial use, distribution, and reproduction in any medium, provided the original author(s) and source are credited.

\section{References}

1. Drinfeld, V.: Almost cocommutative Hopf algebras. Algebra i Analiz 1(2), 30-46 (1989) (Russian) [translation in Leningrad Math. J. 1(2), 321-342 (1990)]

2. Geer, N., Patureau-Mirand, B., Turaev, V.: Modified quantum dimensions and re-normalized link invariants. Compos. Math. 145(01), 196-212 (2009). math.QA/0711.4229

3. Ohtsuki, T.: Quantum invariants. A study of knots and 3-manifolds. Series on Knots and Everything, vol. 29. World Scientific Publishing Co., Inc., River Edge (2002)

4. Reshetikhin, N., Turaev, V.: Ribbon graphs and their invariants derived from quantum groups. Commun. Math. Phys. 127(1), 1-26 (1990) 